\title{
Epidemiological Characteristics of Diabetes Mellitus in Beilun District of Ningbo, China (2012-2018)
}

\author{
Zhouyang Le' ${ }^{1}$, Qunxiong Hu², Kuihua Tong³, Chenghan Zhou ${ }^{3}$, Jinjun Li ${ }^{*}$ \\ ${ }^{1}$ College of Medicine, Wuhan University of Science and Technology, Wuhan, China \\ ${ }^{2}$ Beilun District Disease Prevention and Control Center Ningbo, China \\ ${ }^{3}$ The Second People's Hospital of Beilun District, Ningbo, China \\ Email: *entry2003@126.com
}

How to cite this paper: Le, Z.Y., Hu, Q.X., Tong, K.H., Zhou, C.H. and Li, J.J. (2020) Epidemiological Characteristics of Diabetes Mellitus in Beilun District of Ningbo, China (2012-2018). Journal of Diabetes Mellitus, 10, 98-107.

https://doi.org/10.4236/jdm.2020.102009

Received: April 10, 2020

Accepted: May 16, 2020

Published: May 19, 2020

Copyright $\odot 2020$ by author(s) and Scientific Research Publishing Inc. This work is licensed under the Creative Commons Attribution International License (CC BY 4.0).

http://creativecommons.org/licenses/by/4.0/

\section{(c) (i) Open Access}

\begin{abstract}
Aim: To explore the trend of diabetes epidemic characteristics in Beilun District of Ningbo, China and to provide basis for further improvement and improvement of diabetes prevention and treatment program. Methods: The data of chronic disease monitoring information management system in Zhejiang Province were collected, and the data of diabetes monitoring in Beilun District of Ningbo, China from 2012 to 2018 were compared and analyzed. Results: Compared with 2012-2015 years, the incidence rate of diabetes in this area was well controlled in 2016-2018 years, from 738.35/100 thousand to $538.27 / 100$ thousand $(\mathrm{P}<0.05)$. The proportion of type 2 diabetes decreased from $88.36 \%$ to $83.09 \%(\mathrm{P}<0.05)$. Among them, the increase of diabetes incidence rate was controlled in the $20-39$ year old age group. The incidence rate of diabetes increased again in the 10 - 19 year old group, while the proportion of gestational diabetes mellitus was still on the rise. The proportion of people with diabetes mellitus with Abnormal BMI (body mass index) increased year by year. Conclusion: The incidence rate of diabetes in this region is related to the preferences of diet and lifestyle in 2012-2018 years. Among them, BMI is a risk factor for many chronic diseases. The incidence rate of BMI abnormal diabetes mellitus is increasing year by year, and some preventive measures need to be taken.
\end{abstract}

\section{Keywords}

Diabetes Mellitus, Incidence Rate, Lifestyle, Body Mass Index

\section{Background}

With the rapid development of national economy, the living standard of human 
beings has been greatly improved, and the disease spectrum and health concept have changed accordingly. Chronic diseases (NCDs) have gradually become one of the public health problems that seriously threaten human health [1]. With the change of life style, diabetes has developed into one of the chronic diseases that seriously harm the health of residents. Diabetes mellitus (DM) is a common chronic non infectious disease, which can make the body continuously hyperglycemia and long-term metabolic disorder, and lead to the damage, dysfunction and failure of the whole body tissues and organs, especially the eyes, kidneys, cardiovascular system and nervous system. Severe cases may cause acute complications such as dehydration, electrolyte disorder, acid-base imbalance, ketoacidosis, hypertonic coma and other serious complications [2]. In order to explore the trend of diabetes epidemic characteristics in Beilun District of Ningbo, China and to provide scientific reference for future prevention and intervention program modification, the monitoring data of diabetes in Beilun District of Ningbo, China from 2012 to 2018 were analyzed.

\section{Materials and Methods}

\subsection{Data}

The data of this analysis are collected from the chronic disease monitoring information management system of Zhejiang Province from 2012 to 2018. The population data comes from the population statistics of Beilun District Public Security Bureau and civil affairs department. The inclusion criteria of the subjects were: 1) registered residents of Beilun District, Ningbo, China; 2) incidence data: newly diagnosed cases of diabetes; 3 ) onset time of diabetes patients was between 2012 and 2018. The exclusion criteria are: residents living in Beilun District without Beilun household registration.

\subsection{Data Collection}

The collection of new cases of diabetes in Beilun District mainly comes from the chronic disease case reports found and treated by medical and health institutions (including general hospitals, specialized hospitals, private and private hospitals, community health service centers, township hospitals, etc.); when examining the death card, if the existing cases of diabetes need to be reported, they are not reported after checking with the chronic disease report registration system, It is necessary to supplement the corresponding morbidity report card and report the cases found through the missing report investigation and other special investigations. The diagnosis of diabetes is based on the diagnostic criteria of who in 1999 [3]. All reported information shall be reviewed by the center for Disease Control and prevention of Beilun District, Ningbo, China, and duplicate information shall be eliminated.

\subsection{Statistical}

The data were derived from the "network direct report system of chronic diseas- 
es", and were analyzed and sorted out preliminarily with excel2016 software. The years 2012-2015 and 2016-2018 were divided into two control groups. Through SPSS17.0 statistical software for statistical analysis, $\mathrm{P}<0.05$ showed that the difference was statistically significant

\section{Results}

\subsection{Years Report on Incidence Rate and Incidence of Diabetes Mellitus}

The incidence rate of diabetes in Beilun residents in the 2012-2018 years was $878.00 / 100$ thousand, 774.23/100k, 639.12/100k, 665.80/100k, 529.97/100k, $597.09 / 100 \mathrm{k}, 488.86 / 100 \mathrm{k}$. There were 7718 cases of male diabetes and 9057 cases of female diabetes. The ratio of male to female was $0.85: 1$. The incidence rate of male diabetes was $606.48 / 100 \mathrm{k}$, and female $691.51 / 100 \mathrm{k}$. The age of onset of diabetes was 0 - 98 years old $(55.31 \pm 16.24)$ years on average. The age of onset of diabetes in men $(58.88 \pm 13.50)$ years old was later than that in women $(52.31 \pm$ 17.68). The age difference between men and women was statistically significant $(\mathrm{t}=20.644, \mathrm{P}<0.05)$. See Table 1 for details.

\subsection{Age Specific Morbidity}

The age of onset of diabetes mellitus shows a trend of low age, the most obvious increase is in the age group of 10 - 19 years old, and the trend of increase appears again after being controlled in 12 - 15 years. The incidence rate of diabetes in 40 - 69 years old has been effectively controlled. Due to the small number of cases in the age group of 0 - 9 years old, there is a large random error, but we can't despise the intervention and prevention of diabetes in children, especially in newborns. For specific data on incidence rate of diabetes in different ages and ages, see Table 2.

\subsection{Composition of Disease Types}

The incidence of diabetes in Beilun District is mainly type II diabetes, and the

Table 1. Diabetes incidence rate in Beilun district in 2012-2018 (/100 thousand).

\begin{tabular}{|c|c|c|c|c|c|c|c|c|}
\hline \multirow{2}{*}{ year } & \multicolumn{3}{|c|}{ Population } & \multicolumn{3}{|c|}{ incidence rate } & \multirow{2}{*}{$x^{2}$} & \multirow{2}{*}{$\mathrm{P}$} \\
\hline & sum & male & female & incidence rate & Incidence rate of men & Incidence rate of women & & \\
\hline 2012 & 354,213 & 175,671 & 178,542 & $878.00(3110)$ & $807.76(1419)$ & $947.12(1691)$ & 19.759 & 0.000 \\
\hline 2013 & 357,001 & 176,755 & 180,246 & $774.23(2764)$ & $749.63(1325)$ & 798.35 (1439) & 2.758 & 0.097 \\
\hline 2014 & 360,496 & 178,224 & 182,273 & $639.12(2304)$ & $593.08(1057)$ & $684.14(1247)$ & 11.768 & 0.001 \\
\hline 2015 & 365,276 & 180,262 & 185,014 & $665.80(2432)$ & $628.53(1133)$ & 702.11 (1299) & 7.474 & 0.006 \\
\hline 2016 & 372,095 & 183,157 & 188,938 & $529.97(1972)$ & $454.81(833)$ & $602.83(1139)$ & 38.665 & 0.000 \\
\hline 2017 & 381,516 & 187,133 & 194,383 & $597.09(2278)$ & $560.55(1049)$ & $632.27(1229)$ & 8.186 & 0.004 \\
\hline 2018 & 391,729 & 191,377 & 200,352 & $488.86(1915)$ & $471.31(902)$ & $505.62(1013)$ & 2.366 & 0.124 \\
\hline Average & 368,904 & 181,797 & 187,107 & $649.49(2396)$ & $606.72(1103)$ & $691.04(1293)$ & 10.162 & 0.001 \\
\hline
\end{tabular}


Table 2. 2014-2018 year diabetes incidence rate in Beilun district (/100 thousand).

(a)

\begin{tabular}{ccccccccccc}
\hline Year/age & $0-9$ & $10-19$ & $20-29$ & $30-39$ & $40-49$ & $50-59$ & $60-69$ & $70-79$ & $\geq 80$ \\
\hline 2012 & 7.55 & 37.13 & 361.33 & 405.30 & 773.36 & 1256.69 & 2154.16 & 2478.60 & 1908.51 \\
2013 & 10.84 & 14.10 & 429.32 & 407.88 & 706.02 & 1262.49 & 1554.37 & 1719.90 & 1602.51 \\
2014 & 0.00 & 13.97 & 423.21 & 395.02 & 528.00 & 996.67 & 1265.61 & 1421.27 & 1367.71 \\
2015 & 6.47 & 18.86 & 440.72 & 432.62 & 557.44 & 1006.75 & 1225.76 & 1408.27 & 1172.88 \\
$12-15$ & 6.21 & 21.01 & 413.65 & 410.21 & 641.21 & 1130.65 & 1549.98 & 1757.01 & 1512.90 \\
2016 & 11.90 & 7.49 & 403.39 & 366.93 & 376.29 & 706.62 & 1040.72 & 1163.49 & 1019.50 \\
2017 & 0.00 & 17.84 & 409.93 & 448.26 & 431.10 & 753.53 & 1137.98 & 1486.54 & 1143.48 \\
2018 & 2.41 & 20.20 & 377.30 & 374.04 & 331.38 & 703.52 & 910.60 & 1077.16 & 784.84 \\
$16-18$ & 4.77 & 15.18 & 396.87 & 396.41 & 379.59 & 721.22 & 1029.76 & 1242.39 & 982.61 \\
Intergroup growth & $-23.19 \%$ & $-2.77 \%$ & $-4.04 \%$ & $-3.36 \%$ & $-40.80 \%$ & $-36.21 \%$ & $-33.56 \%$ & $-29.29 \%$ & $-35.05 \%$ \\
\hline
\end{tabular}

(b)

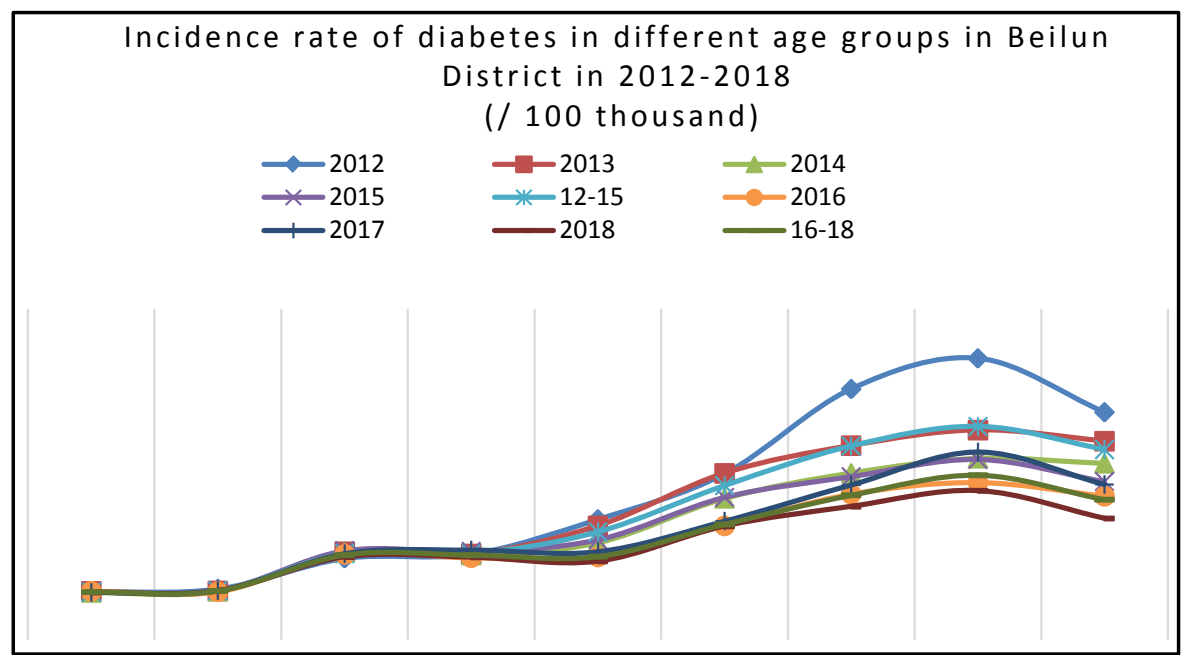

number of men is more than that of women. The incidence of gestational diabetes increased significantly. See Table 3 for details.

\subsection{Incidence Rate of Type II Diabetes at Different Ages}

Type II diabetes accounted for $86.41 \%$ of the total cases of diabetes from 2012 to 2018, and was the main body of the population with diabetes. Among them, male accounted for $46.05 \%$ and female accounted for $40.36 \%$. There was significant difference between male and female $\left(\chi^{2}=93.963, \mathrm{P}<0.05\right)$. See Table 4 for details.

\subsection{BMI of People with Diabetes}

The BMI of the diabetic population tends to be overweight and obesity. BMI = the square of body weight/height (international unit: $\mathrm{kg} / \mathrm{m}^{2}$ ), the standard shall refer to the Chinese reference standard. See Table 5 for details. 
Table 3. Composition of disease types of diabetes in Beilun district from 2014 to 2018 (male/female).

(a)

\begin{tabular}{|c|c|c|c|c|c|}
\hline year & Type I diabetes & Type II diabetes & Gestational diabetes & Special diabetes & Total \\
\hline 2012 & $0.48 \%(15) / 0.51 \%(16)$ & $48.50 \%(1508) / 43.03 \%(1338)$ & $0.00 \%(0) / 6.88 \%(214)$ & $0.33 \%(10) / 0.27 \%(8)$ & $100.00 \%(3110)$ \\
\hline 2014 & $0.09 \%(2) / 0.00 \%(0)$ & $45.66 \%(1052) / 39.88 \%$ (919) & $0.00 \%(0) / 14.06 \%(324)$ & $0.13 \%(3) / 0.18 \%(4)$ & $100.00 \%(2304)$ \\
\hline 2015 & $0.25 \%(6) / 0.29 \%(7)$ & $45.97 \%(1118) / 40.13 \%(976)$ & $0.00 \%(0) / 12.82 \%(312)$ & $0.37 \%(9) / 0.17(4)$ & $100.00 \%(2432)$ \\
\hline $12-15$ & $0.26 \%(28) / 0.25 \%(27)$ & $46.98 \%(4983) / 41.38 \%(4390)$ & $0.00 \%(0) / 10.58 \%(1123)$ & $0.33 \%(35) / 0.22 \%(23)$ & $100.00 \%(10610)$ \\
\hline 2016 & $0.41 \%(8) / 0.20 \%(4)$ & $41.58 \%(820) / 41.02 \%$ (809) & $0.00 \%(0) / 16.13 \%(318)$ & $0.25 \%(5) / 0.41 \%(8)$ & $100.00 \%(1972)$ \\
\hline 2017 & $0.26 \%(6) / 0.31 \%(7)$ & $45.52 \%(1037) / 38.59 \%(879)$ & $0.00 \%(0) / 14.84 \%(338)$ & $0.26 \%(6) / 0.22 \%(5)$ & $100.00 \%(2278)$ \\
\hline 2018 & $0.57 \%(11) / 0.52 \%(10)$ & $46.22 \%(885) / 36.14 \%(692)$ & $0.00 \%(0) / 16.03 \%(307)$ & $0.31 \%(6) / 0.21 \%(4)$ & $100.00 \%(1915)$ \\
\hline Sum & $0.32 \%(53) / 0.29 \%(48)$ & $46.05 \%(7725) / 40.36 \%(6770)$ & $0.00 \%(0) / 12.43 \%(2086)$ & $0.31 \%(52) / 0.24 \%(40)$ & $100.00 \%(16,775)$ \\
\hline
\end{tabular}

(b)



Table 4. 2014-2018 age incidence rate of type II diabetes in Beilun area (male/female; /100 thousand).

\begin{tabular}{ccccccccccccccc}
\hline Year/age & $0-9$ & $10-19$ & $20-29$ & $30-39$ & $40-49$ & $50-59$ & $60-69$ & $70-79$ \\
\hline 2012 & $0.00 / 0.00$ & $19.46 / 13.25$ & $74.46 / 64.51$ & $207.36 / 160.42$ & $667.46 / 531.54$ & $1253.63 / 997.51$ & $1653.44 / 1466.57$ & $1648.53 / 1701.32$ & $1865.14 / 1658.21$ \\
2013 & $0.00 / 0.00$ & $15.33 / 16.49$ & $72.65 / 54.20$ & $215.45 / 130.16$ & $594.55 / 486.16$ & $1164.55 / 967.62$ & $1465.14 / 1265.15$ & $1543.14 / 1648.55$ & $1684.15 / 1311.19$ \\
2014 & $0.00 / 0.00$ & $6.88 / 7.09$ & $68.02 / 42.23$ & $203.86 / 119.78$ & $588.61 / 456.24$ & $1049.52 / 934.16$ & $1369.53 / 1152.95$ & $1335.65 / 1498.76$ & $1559.50 / 1177.22$ \\
2015 & $0.00 / 0.00$ & $22.16 / 15.43$ & $78.67 / 71.24$ & $220.06 / 178.75$ & $646.63 / 455.89$ & $1122.85 / 865.73$ & $1241.73 / 1197.75$ & $1385.81 / 1401.48$ & $1362.60 / 1013.17$ \\
$12-15$ & $0.00 / 0.00$ & $14.25 / 11.08$ & $73.11 / 56.10$ & $212.14 / 150.02$ & $624.73 / 496.08$ & $1187.53 / 948.90$ & $1401.16 / 1277.10$ & $1462.24 / 1547.95$ & $1675.16 / 1391.32$ \\
2016 & $0.00 / 0.00$ & $14.65 / 0.00$ & $58.09 / 76.65$ & $135.84 / 66.06$ & $408.42 / 316.62$ & $725.04 / 672.24$ & $983.38 / 1080.61$ & $1142.13 / 1157.34$ & $1030.17 / 982.77$ \\
2017 & $0.00 / 0.00$ & $13.85 / 0.00$ & $49.07 / 74.15$ & $212.24 / 121.60$ & $530.38 / 296.24$ & $839.51 / 660.77$ & $1170.35 / 1065.96$ & $1488.65 / 1375.76$ & $1301.86 / 988.58$ \\
2018 & $0.00 / 0.00$ & $13.10 / 13.86$ & $56.46 / 38.46$ & $188.95 / 71.94$ & $473.95 / 165.02$ & $746.29 / 634.87$ & $933.77 / 848.29$ & $1179.48 / 936.39$ & $831.80 / 732.90$ \\
$16-18$ & $0.00 / 0.00$ & $13.84 / 4.84$ & $54.62 / 63.77$ & $179.77 / 83.48$ & $470.04 / 259.40$ & $769.93 / 655.94$ & $1029.44 / 995.51$ & $1270.63 / 1181.78$ & $1051.84 / 899.36$ \\
\hline
\end{tabular}


Table 5. BMI composition of diabetic population in Beilun District from 2014 to 2018.

\begin{tabular}{ccccc}
\hline Year & normal weight or lean & (overweight) & $\geq 27$ (Obesity) & Total \\
\hline 2012 & $1919(61.70 \%)$ & $971(31.23 \%)$ & $220(7.07 \%)$ & $3110(100.00 \%)$ \\
2013 & $1669(60.37 \%)$ & $899(32.51 \%)$ & $196(7.12 \%)$ & $2764(100.00 \%)$ \\
2014 & $1347(58.46 \%)$ & $783(33.99 \%)$ & $174(7.55 \%)$ & $2304(100.00 \%)$ \\
2015 & $1459(59.99 \%)$ & $779(32.03 \%)$ & $194(7.98 \%)$ & $2432(100.00 \%)$ \\
$12-15$ & $6394(60.26 \%)$ & $3432(32.35 \%)$ & $784(7.39 \%)$ & $10,610(100.00 \%)$ \\
2016 & $1071(54.31 \%)$ & $699(35.45 \%)$ & $202(10.24 \%)$ & $1972(100.00 \%)$ \\
2017 & $1222(53.64 \%)$ & $833(36.57 \%)$ & $223(9.79 \%)$ & $2278(100.00 \%)$ \\
2018 & $989(51.65 \%)$ & $686(35.82 \%)$ & $240(12.53 \%)$ & $1915(100.00 \%)$ \\
$16-18$ & $3282(53.23 \%)$ & $2218(35.98 \%)$ & $665(10.79 \%)$ & $6165(100.00 \%)$ \\
sum & $6088(55.85 \%)$ & $3780(34.67 \%)$ & $1033(9.48 \%)$ & $10,901(100.00 \%)$ \\
\hline
\end{tabular}

\subsection{Changes of Diabetes Epidemic Characteristics in 2012-2015 and 2016-2018}

\subsubsection{Incidence Rate Changes}

The incidence rate of 10,610 cases was 2012-2015 years, the average incidence rate was 738.35/100 thousand, and the total incidence of 6165 cases in 2016-2018 years, the average incidence rate was 538.27/100 thousand. Compared with $2012-2015$, the incidence rate was significantly reduced $\left(\chi^{2}=31.549, \mathrm{P}<0.05\right)$, and the annual incidence rate of diabetes was well controlled.

\subsubsection{Age Specific Incidence Rate Changes}

Compared with 2012-2015 years, 2016-2018 years can see that intervention and prevention and treatment effect is more significant, the two groups have achieved negative growth contrast, the incidence rate of diabetes in the main population of $40-49$ years has been well controlled $(-40.80 \%)$. Although the incidence rate incidence rate of 10 - 39 years old was controlled to a certain extent, the negative growth trend was significantly lower than that of other groups. The incidence rate of diabetes in the 10 - 19 year old group increased continuously in $16-18$ years, suggesting that the control of diabetes incidence in the $10-19$ year old population in the region needs more attention. Through literature review, we can see that the awareness rate of diabetes in the age group of 10 - 19 years old is only $50 \%$, and there are more than $80 \%$ adverse lifestyle, such as drinking drinks and eating fried food, etc., and the awareness of obesity and diabetes is insufficient [4].

\subsubsection{Changes in the Composition of Diabetic Disease Types}

Compared with 2012-2015, the incidence of type II diabetes in 2016-2018 has been well controlled, and other groups have increased slightly, especially gestational diabetes.

3.6.4. Incidence Rate of 2.6.4 Type II Diabetes Reported at Different Ages Diabetes was not detected in the $0-9$ age group. The incidence rate of incidence 
of all age groups was lower than that of 2012-2015 years, except for 20 - 29 years. The incidence rate of all age groups decreased. In addition, the incidence of chi square test showed that the difference between male and female groups was statistically significant in 30 - 69 years of age group and 80 years old and above. The chi square test showed that the incidence rate of female age group was significantly different from that of the $20-29$ age group.

\subsubsection{Changes of BMI Composition in Diabetic Population}

Compared with 2012-2015, in 2016-2018, the proportion of normal and lean people decreased significantly, while the proportion of obese people increased significantly. BMI, as a risk factor related to diabetes, needs special attention.

\section{Discussion}

In recent years, with the improvement of living standards and the change of eating habits, bad eating and living habits are eroding the health of residents. The incidence rate of incidence of chronic diseases caused by aging population is increasing. As a coastal city with a good economic development and good living standard, the incidence rate of diabetes in Beilun District of Ningbo, China has always been higher than the average level of the whole city.

The incidence rate of diabetes in Beilun District of Ningbo, China was reduced from 738.35/100 thousand in 2012-2015 years to 538.27/100 thousand in 2016-2018 years, which was well controlled. This is closely related to the prevention and treatment of diabetes through multi sector collaboration in Beilun district. The incidence rate of diabetes reported in the age group 40 and above is the best. The trend of diabetes onset is controlled to a certain extent. The incidence rate of diabetes in the $10-19$ year old group has been increased for three consecutive years after being controlled. This may be related to the improvement of teenagers' living conditions, the long-term high sugar and fat intake brought about by their enthusiasm for taking out food service, and the unhealthy living habits such as irregular work and rest and lack of exercise for a long time. Studies have shown that long-term high-energy diet is a major cause of the rapid rise in diabetes incidence rate. [5], the same long-term consumption of red meat and processed meat products can lead to decreased insulin sensitivity and increase the risk of diabetes [6]. It is an effective way to improve the condition of diabetes by changing the diet structure. Many studies have shown that the Mediterranean diet, which is mainly composed of grains, vegetables, fish, milk, fruits and olive oil, has great potential in blocking the occurrence and development of diabetes [7], which may be related to vitamin $\mathrm{D}$, calcium and magnesium ions in milk and a large number of unsaturated fatty acids in olive oil and fish. At the same time, mate analysis showed that vegetarianism was negatively correlated with the risk of diabetes [8]. Even after the correction of body mass index (BMI), the incidence rate of diabetes in meat eating people is 2 times that of vegetarians. The prevalence of type 2 diabetes in vegetarians and vegetarians was $77 \%$ and $54 \%$ lower than that in non vegetarians [9]. After the full liberali- 
zation of the two child policy, the proportion of gestational diabetes between 2016-2018 and 2012-2015 has not been effectively controlled. Among them, there are factors such as the increase in the number of pregnant women. I think the main reason for the increase is the change of diet and living habits and the increase of life pressure. Some studies have shown that the abnormal increase of blood glucose during pregnancy will easily affect the brain development, learning and memory of the fetus, and also increase the risk of cardiovascular disease in the adult fetus. In the prevention and health care work for pregnant women, we should monitor the blood glucose concentration of pregnant women who have gestational diabetes risk, and increase the health diet of pregnant women, effectively improve diet structure and develop good habits, so as to reduce the incidence rate of gestational diabetes mellitus. The occurrence of gestational diabetes mellitus in pregnant women and the change of the environment in early neonatal development may be an inducement factor for the increase of the incidence rate of type 0 - 9 diabetes in children. Body mass index (BMI) is not only an independent, dose-dependent risk factor for diabetes [10], but also a related risk factor for a variety of chronic diseases. The proportion of people with $\mathrm{Ab}$ normal BMI in the population with diabetes in Beilun District has increased year by year. Compared with 2012-2015, the proportion of obese people has increased significantly in 2016-2018. It has been found that the proportion of diabetes patients with high salt diet and high fat diet in China of Ningbo is relatively high, while the number of people taking part in physical training is relatively small. Nearly two-thirds of people travel by means of transportation, and the amount of exercise is relatively insufficient [11]. Because the drug intervention needs a lot of capital and human investment, the current lifestyle intervention is the main way of community intervention [12]. At the same time, studies have shown that after three months of intensive intervention including reasonable diet and appropriate exercise, the physiological indexes of blood glucose, blood pressure, blood lipid and other factors that lead to the increased risk of type II diabetes have improved significantly, which is significantly lower than that of the control group and has statistical significance [13]. Only through regular follow-up, the contracted service form of family doctors is adopted, i.e. outpatient doctors provide 1 . At the end of each month, the assistant doctors combined with the outpatient doctors' diagnosis and treatment services to carry out follow-up life style intervention for 8 months, the effect is not good. Because the intervention time is short, it is difficult to completely change the unhealthy life habits and bad behavior habits of patients developed for a long time, and only to a certain extent at the beginning of the intervention, then only to maintain the patients. There was no further improvement in the health level of [14]. It can be seen that the treatment of long-term lifestyle intervention is effective for the treatment and prevention of diabetes.

In order to reduce the incidence rate of diabetes effectively, we need to carry out diabetes related business guidance for primary health care units, promote diabetes prevention and control technologies, popularize diabetes related know- 
ledge, and improve the level of diagnosis and treatment of diabetes management doctors. On the other hand, we should strengthen the prevention and treatment of high-risk individuals, and strengthen the publicity and popularization of diabetes related knowledge in communities and schools, actively guide healthy lifestyle development, raise awareness of the risk factors of diabetes, and enhance the awareness of physical examination among the masses, so as to control the incidence rate.

\section{Conflicts of Interest}

The authors declare no conflicts of interest regarding the publication of this paper.

\section{References}

[1] Mishel, M.H. and Braden, C.J. (1988) Finding Meaning: Antecedents of Uncertainty in Illness. Nursing Research, 37, 98. https://doi.org/10.1097/00006199-198803000-00009

[2] Cheng Alice, Y.Y. (2013) Canadian Diabetes Association 2013 Clinical Practice Guidelines for the Prevention and Management of Diabetes in Canada. Introduction. Canadian Journal of Diabetes, 37, S1-S3. https://doi.org/10.1016/j.jcjd.2013.04.005

[3] Qian, R.L. (2000) New Diagnostic Criteria and Classification of Diabetes. Chinese Journal of Diabetes, No. 1, 4-5.

[4] Lu, X.Y., Xiao, Y.Y., Wang, J., et al. (2018) Survey of Life Style and Diabetes Awareness Rate of Primary and Secondary School Students in Shanghai Suburbs. China Medical Journal, 15, 46-49.

[5] Chatterjee, S., Khunti, K. and Davies, M.J. (2017) Type 2 Diabetes. The Lancet, 389, 2239-2251. https://doi.org/10.1016/S0140-6736(17)30058-2

[6] Kim, Y., Keogh, J.B. and Clifton, P.M. (2017) Consumption of Red and Processed Meat and Refined Grains for 4 Weeks Decreases Insulin Sensitivity in Insulin-Resistant Adults: A Randomized Crossover Study. Metabolism, 68, 173-183. https://doi.org/10.1016/j.metabol.2016.12.011

[7] Jannasch, F., Kröger, J. and Schulze, M.B. (2017) Dietary Patterns and Type 2 Diabetes: A Systematic Literature Review and Meta-Analysis of Prospective Studies. The Journal of Nutrition, 147, 1174-1182. https://doi.org/10.3945/jn.116.242552

[8] Lee, Y. and Park, K. (2017) Adherence to a Vegetarian Diet and Diabetes Risk: A Systematic Review and Meta-Analysis of Observational Studies. Nutrients, 9, 603. https://doi.org/10.3390/nu9060603

[9] Melina, V. (2016) Position of the Academy of Nutrition and Dietetics: Vegetarian Diets. Journal of the Academy of Nutrition and Dietetics, 116, 1970-1980. https://doi.org/10.1016/j.jand.2016.09.025

[10] Zhu, Y., Shi, J.H., Luo, X., et al. (2017) Analysis of Dose-Response Relationship between BMI and Diabetes. China Health Statistics, 34, 887-890 + 895.

[11] Lu, H.C., Lin, H.B., Ding, K., et al. (2013) Survey on Prevalence and Risk Factors of Type 2 Diabetes in Yinzhou District. Zhejiang Preventive Medicine, 25, 31-32 + 36.

[12] Li, Y.L., Liu, J., Jing, Y.Q., et al. (2014) Evaluation on the Effect of Intensive Lifestyle Intervention in High Risk Individuals with Type 2 Diabetes. New World of Di- 
abetes, No. 20, 69-70.

[13] Wang, L.Q., Zhu, H.F. and Pan, Q. (2016) Survey of Impaired Glucose Regulation and Prevalence of Type 2 Diabetes in Community and Effect of Intensive Lifestyle Intervention. Chinese Journal of Gerontology, 36, 2763-2765.

[14] Yu, X.L., Zhang, L.J., Zhang, J., et al. (2017) Evaluation on the Service Effect of Community Family Doctors in Patients with Type 2 Diabetes. Preventive Medicine, 29, 472-474. 